N.-R. Shieh

Nagoya Math. J.

Vol. 122 (1991), 1-17

\title{
WHITE NOISE ANALYSIS AND TANAKA FORMULA FOR INTERSECTIONS OF PLANAR BROWNIAN MOTION
}

\author{
NARN-RUEIH SHIEH
}

\section{$\S 1$. Introduction}

In this paper, we shall use Hida's [5, 7, 9] theory of generalized Brownian functionals (or named white noise analysis) to establish a stochastic integral formula concerning the multiple intersection local times of planar Brownian motion $B(t)$. First, we review some basic facts. It is well-known that, for each $r \geqslant 2$, a.s. $B(t)$ has points of multiplicity $r$, see Dvoretzky-Erdös-Kakutani [1] and Geman-Horowitz-Rosen [4]. A formal measure on such $r$-fold self-intersections is

$$
\int_{D} \ldots \int_{D} \delta\left(B\left(t_{2}\right)-B\left(t_{1}\right)\right) \cdots \delta\left(B\left(t_{r}\right)-B\left(t_{r-1}\right)\right) d t_{1} \cdots d t_{r}
$$

where $D \subset\left\{\left(t_{1}, \cdots, t_{r}\right) \mid 0 \leqslant t_{1} \leqslant t_{2} \leqslant \cdots \leqslant t_{r}<\infty\right\}$. Rosen [15] proved that (a.s.) there exists measurable $a_{r}(x, D), x \in R^{2(r-1)}$, called the $r$-fold intersection local time, such that for all bounded measurable $g(x)$ on $R^{2(r-1)}$

$$
\begin{gathered}
\int \underbrace{}_{D} \int g\left(B\left(t_{2}\right)-B\left(t_{1}\right), \cdots, B\left(t_{r}\right)-B\left(t_{r-1}\right)\right) d t_{1} \cdots d t_{r} \\
=\int_{R^{2(r-1)}} \cdots \int g(x) \alpha_{r}(x, D) d x .
\end{gathered}
$$

In case $D=\prod_{i=1}^{r}\left[a_{i}, b_{i}\right], 0<a_{1}<b_{1}<a_{2}<b_{2}<\cdots<b_{r}$ (the off-diagonal case), Rosen [15,17] proved that (a version of) $\alpha_{r}(x, D)$ can be chosen so that it is (a.s.) jointly continuous in $x, a_{i}, b_{i}$. Thus, (1.1) is represented as $\alpha_{r}(0, D)$ in this case. However, in case $D=D_{T}=\left\{\left(t_{1}, \cdots, t_{r}\right) \mid 0 \leqslant t_{1} \leqslant \cdots\right.$ $\left.t_{r} \leqslant T\right\}$ (the diagonal case), then $\alpha_{r}(x, T) \equiv \alpha_{r}\left(x, D_{T}\right)$ can only be continuous on $\left(R^{2} \backslash\{0\}\right)^{r-1}$. To study the asymptotics of $\alpha_{r}(x, T)$ as $x \rightarrow 0$, it is intended to find that after substracting off certain explicit "infinite part" from $\alpha_{r}(x, T)$ the remainder $\tilde{\alpha}_{r}(x, T)$, called the renormalized intersection local time, admits a jointly continuous extension to all $(x, T) \in R^{2(r-1)} \times R_{+}$.

Received December 3, 1988. 
The research was initiated by Varadhan [22] and has been studied intensively by Dynkin, LeGall, Rosen and Yor, see Dynkin [2]. In the investigations Tanaka type formulae for $\alpha_{r}$ and $\tilde{\alpha}_{r}$, analogous to the classical Tanaka's formula for one-dimensional Brownian local time, play an important role. In the off-diagonal case, let $\alpha_{r}\left(x, a_{1}, \cdots, a_{r}, d t_{r}\right), t_{r} \in\left[a_{r}, b_{r}\right]$, denote the Borel measure induced by $t_{r} \rightarrow \alpha_{r}\left(\cdots, t_{r}\right)$ and $\alpha_{1}\left(d t_{1}\right) \equiv d t_{1}$, the Lebesgue measure on $R^{1}$. The Tanaka formulae relating $\alpha_{r+1}$ and $\alpha_{r}$ were studied by Rosen [16, 17] and Yor [23]. Then, the formulae were used by them $[18,20,23]$ to find the explicit forms of $\tilde{\alpha}_{2}$ and $\tilde{\alpha}_{3}$.

In this paper, we shall prove the following formula, which is an extension of Yor [23] from $r=1$ to general $r \geqslant 1$. It corresponds also to Rosen [17], who used the potential kernel for the killed Brownian motion instead of $\log |x|$.

Theorem 1.1. Assume that $0<a_{i}<b_{i}<a_{i+1}<b_{i+1}, i=1, \cdots, r$. For each $x=\left(x_{1}, \cdots, x_{r-1}\right) \in R^{2(r-1)}$ and $x_{r} \in R^{2}$ the following equality holds almost surely.

$$
\begin{aligned}
& \int_{a_{r}}^{b_{r}} \log \left|B\left(b_{r+1}\right)-B\left(t_{r}\right)-x_{r}\right| \alpha_{r}\left(x, a_{1}, \cdots, a_{r}, d t_{r}\right) \\
& \quad-\int_{a_{r}}^{b_{r}} \log \left|B\left(a_{r+1}\right)-B\left(t_{r}\right)-x_{r}\right| \alpha_{r}\left(x, a_{1}, \cdots, a_{r}, d t_{r}\right) \\
&=\int_{a_{r+1}}^{b_{r+1}}\left(\int_{a_{r}}^{b_{r}} \frac{B\left(t_{r+1}\right)-B\left(t_{r}\right)-x_{r}}{\left|B\left(t_{r+1}\right)-B\left(t_{r}\right)-x_{r}\right|^{2}} \alpha_{r}\left(x, a_{1}, \cdots, a_{r}, d t_{r}\right)\right) \cdot d B\left(t_{r+1}\right) \\
& \quad+\pi \alpha_{r+1}\left(\left(x, x_{r}\right), a_{1}, \cdots, a_{r+1}, b_{r+1}\right) ;
\end{aligned}
$$

in the above, the integrand for the (Ito's) stochastic integral will be proved to be the $L^{2}\left(d t_{\tau+1} \times d P\right)$ limit of certain approximation sequence.

Our formula will suggest an explicit form for $\tilde{\alpha}_{r}$, which is an extension of Yor [23, $r=2$ ] and Rosen-Yor [20, $r=3$ ] to general $r$. See $\S 5$.

We explain our viewpoint on using white noise analysis to derive (1.2). Kubo [11] established an Ito formula for generalized Brownian functionals and indicated briefly to view the classical Tanaka's formula as a case of his result [11, Remark 4.1]. Thus, we are motivated to derive (1.2) from Kubo's formula. The crucial point is that Kubo's formula holds in Hida's space $\left(L^{2}\right)^{-}$, while our (1.2) is an a.s. equality. Therefore, in order to achieve our formula we must "realize" a Brownian functional from generalized one to ordinary one. Our procedure in based on or- 
thogonal series expansions of generalized Brownian functionals. The expansions are known to hold in Hida's space $\left(L^{2}\right)^{-}$, and we carry out precise computations to show that the expansions are in fact convergent in $L^{2}$ sense. In a previous work Kuo-Sheih [13], Yor's result (i.e. (1.2) with $r=1$ ) was reproved by using realization techniques. However, the setting in $[13, \S 4]$ is improper, because of the doubtful definition $f(B(t, \omega), \omega)$ given there.

The remaining part of this paper will be devided into four sections. In $\S 2$, we shall summarize briefly Hida's theory. In $\S 3$, we shall prove two realization results of generalized Brownian functionals. Then, we shall prove a Tanaka type formula in $\S 4$ and derive Theorem 1.1. In the final $\S 5$, we shall discuss how to apply (1.2) to obtain an explicit form of the renormalized $\tilde{\alpha}_{r}$.

The present work was initiated while the author visited Nagoya University in August, 1988. The hospitality of the Mathematical Department and the inspiring discussions with Professor T. Hida are deeply appreciated.

\section{§2. Summary on generalized Brownian functionals}

Let $E \subset L^{2}(R) \subset E^{*}$ be a Gel'fand triplet and $\mu$ be the standard Gaussian measure on $E^{*}$, i.e. its characteristic functional is given by $\left.C(\xi)=\exp \left[-\|\xi\|^{2} / 2\right)\right],\|\cdot\|$ being the $L^{2}(R)$ norm. Let $K_{n}, K_{n}^{(n)}, K_{n}^{(-n)}$ consist respectively of the multiple Wiener integrals of symmetric functions in Hilbert space $L^{2}\left(R^{n}\right)$, Sobolev space $H^{(n+1) / 2}\left(R^{n}\right)$ and negative Sobolev space $H^{-(n+1) / 2}\left(R^{n}\right)$; see Hida [6]. By Wiener-Ito decomposition theorem, we have

$$
\left(L^{2}\right) \equiv L^{2}\left(E^{*}, \mu\right)=\sum_{n=0}^{\infty} \oplus K_{n} .
$$

Hida [5, 7, 9] introduced the following two spaces

$$
\begin{aligned}
& \left(L^{2}\right)^{+} \equiv \sum_{n=0}^{\infty} \oplus K_{n}^{(n)}, \\
& \left(L^{2}\right)^{-} \equiv \sum_{n=0}^{\infty} \oplus K_{n}^{(-n)},
\end{aligned}
$$

and established the continuous inclusions $\left(L^{2}\right)^{+} \hookrightarrow\left(L^{2}\right) \hookrightarrow\left(L^{2}\right)^{-}$. Members in these three spaces are called respectively test, ordinary and gener. alized Brownian functionals. A calculus has been developed on $\left(L^{2}\right)^{-}$ which is usually referred to white noise analysis or Hida's calculus. To 
visualize an element $\phi \in\left(L^{2}\right)^{-}$, we consider its $\mathscr{S}$-transform (or say $\mathscr{U}$ functional):

$$
(\mathscr{S} \phi)(\xi)=\exp \left(-\frac{\|\xi\|^{2}}{2}\right)\left\langle e^{(\cdot, \xi)}, \phi(\cdot)\right\rangle, \quad \xi \in E ;
$$

$\langle\langle\cdot, \cdot\rangle\rangle$ being the pairing between $\left(L^{2}\right)^{+}$and $\left(L^{2}\right)^{-}$.

Note that $B(t, \omega)=\left(\left\langle\omega, 1_{[0, t]}\right\rangle,\left\langle\omega, 1_{[-t, 0]}\right\rangle\right), t \geqslant 0$ and $\omega \in E^{*}$, is a planar Brownian motion. For $0<t_{1}<t_{2}<\cdots<t_{r}$ and $u_{1}, \cdots, u_{r-1} \in \mathscr{S}^{*}\left(R^{2}\right)$, the space of tempered distributions on $R^{2}$, the formal product

$$
\prod_{i=1}^{r-1} u_{i}\left(B\left(t_{i+1}\right)-B\left(t_{i}\right)\right)
$$

can be defined as a member of $\left(L^{2}\right)^{-}$; its $\mathscr{S}$-transform is given by

$$
\begin{aligned}
\mathscr{P}\left(\prod_{i=1}^{r-1}\right. & \left.u_{i}\left(B\left(t_{i+1}\right)-B\left(t_{i}\right)\right)\right)(\xi) \\
& =\prod_{i=1}^{r-1} \mathscr{S}\left(u_{i}\left(B\left(t_{i+1}\right)-B\left(t_{i}\right)\right)(\xi)\right. \\
& =\prod_{i=1}^{r-1}\left(u_{i} * g_{t_{i+1}-t_{i}}\right)\left(\int_{t_{i}}^{t_{i+1}} \xi(s) d s, \int_{-t_{i+1}}^{-t_{i}} \xi(s) d s\right),
\end{aligned}
$$

see Kubo [11], Kuo [12] and Kallianpur-Kuo [10]. Moreover, the orthogonal series expansion of (2.1) in $\left(L^{2}\right)^{-}$is given by

$$
\begin{aligned}
(2 \pi)^{-r+1} & \prod_{i=1}^{r-1}\left(t_{i+1}-t_{i}\right)^{-1} \sum_{\substack{\left.m_{i=}=n_{i}\right)=(0,0) \\
i=1, \cdots, r-1}} \prod_{\substack{i=1 \\
\text {-1 }}}^{r-1}\left\{\frac{1}{m_{i} ! n_{i} ! 2^{m_{i}} 2^{n_{i}}}\right. \\
& \times\left\langle u_{i}\left(x_{1}, x_{2}\right), H_{m_{i}}\left(\frac{x_{1}}{\sqrt{2\left(t_{i+1}-t_{i}\right)}}\right) H_{n_{i}}\left(\frac{x_{2}}{\sqrt{2\left(t_{i+1}-t_{i}\right)}}\right)\right. \\
& \left.\cdot \exp \left(-\frac{\left(x_{1}^{2}+x_{2}^{2}\right)}{2\left(t_{i+1}-t_{i}\right)}\right)\right\rangle_{\left(x_{1}, x_{2}\right)} \\
& \left.\times H_{m_{i}}\left(\frac{\left\langle\omega, 1_{\left[t_{i}, t_{i+1}\right]}\right\rangle}{\sqrt{2\left(t_{i+1}-t_{i}\right)}}\right) H_{n_{i}}\left(\frac{\left\langle\omega, 1_{\left[-t_{i+1},-t_{i}\right]}\right\rangle}{\sqrt{2\left(t_{i+1}-t_{i}\right)}}\right)\right\} .
\end{aligned}
$$

In the above, $\left\langle u\left(x_{1}, x_{2}\right), \psi\left(x_{1}, x_{2}\right)\right\rangle_{\left(x_{1}, x_{2}\right)}$ denotes the pairing between $u \in$ $\mathscr{S}^{*}\left(R^{2}\right)$ and $\psi \in \mathscr{S}\left(R^{2}\right)$, and $H_{n}(x), x \in R$, is the Hermite polynomial of degree $n$.

For $0<t_{1}<t_{2}$ and $u \in \mathscr{S}^{*}\left(R^{2}\right)$, Kubo [11] established the following formula in $\left(L^{2}\right)^{-}$:

$$
u\left(B\left(t_{2}\right)\right)-u\left(B\left(t_{1}\right)\right)=\int_{t_{1}}^{t_{2}} \partial_{t}^{*}(\nabla u)(B(t)) d t+\frac{1}{2} \int_{t_{1}}^{t_{2}}(\Delta u)(B(t)) d t .
$$

In the above $\partial_{t}^{*}=\left(\partial_{(t, 1)}^{*}, \partial_{(t, 2)}^{*}\right)$ is the adjoint of $\dot{B}(t)$-differentiation operator $\left(\partial_{(t, 1)}, \partial_{(t, 2)}\right)$, see Kubo [11] and Hida [8]. 


\section{§3. Realization of generalized Brownian functionals}

Assuming $0<a_{1}<b_{1}<a_{2}<b_{2}<\cdots<b_{r-1}<a_{r}<b_{r}$, we shall prove

Proposition 3.1. Let $u_{i}, i=1, \cdots, r-1$, be positive tempered distributions on $R^{2}$ (or equivalently, they are positive tempered Radon measures on $\left.R^{2}\right)$. Then

$$
\int_{a_{r}}^{b_{r}} \cdots \int_{a_{1}}^{b_{1} r-1} \prod_{i=1}^{r} u_{i}\left(B\left(t_{i+1}\right)-B\left(t_{i}\right)\right) d t_{1} \cdots d t_{r}
$$

is in $\left(L^{2}\right)$.

Remark. From the arguments in Kallianpur-Kuo [10], it is not diffcult to see that

$$
\left(t_{1}, \cdots, t_{r}\right) \in \prod_{i=1}^{r}\left[a_{i}, b_{i}\right] \rightarrow \prod_{i=1}^{r-1} u_{i}\left(B\left(t_{i+1}\right)-B\left(t_{i}\right)\right) \in\left(L^{2}\right)^{-}
$$

is continuous. Thus, (3.1) exists as a generalized Brownian functional. Proposition 3.1 asserts that it can be "realized" as an ordinary one.

To prove Proposition 3.1, we write the summund in (2.2) as

$$
\prod_{i=1}^{r-1} \phi_{i}\left(m_{i}, n_{i}, t_{i}, t_{i+1}\right), \quad m_{i}, n_{i} \geqslant 0 \text { and } t_{i} \in\left[a_{i}, b_{i}\right] .
$$

Lemma 3.1. (i) If $\left(m_{i}, n_{i}\right) \neq\left(m_{i}^{\prime}, n_{i}^{\prime}\right)$ for some $i$, then

$$
E \prod_{i=1}^{r-1} \phi_{i}\left(m_{i}, n_{i}, t_{i}, t_{i+1}\right) \phi_{i}\left(m_{i}^{\prime}, n_{i}^{\prime}, t_{i}^{\prime}, t_{i+1}^{\prime}\right)=0 .
$$

(ii)

$$
\begin{aligned}
E \prod_{i=1}^{r-1} \phi_{i}\left(m_{i}, n_{i}, t_{i}, t_{i+1}\right) \phi_{i}\left(m_{i}, n_{i}, t_{i}^{\prime}, t_{i+1}^{\prime}\right) \\
\leqslant \text { const. }\left(\frac{\prod_{i=1}^{r-1} m_{i} ! n_{i} !}{\left(\sum_{i=1}^{r-1} m_{i}\right) !\left(\sum_{i=1}^{r-1} n_{i}\right) !\left(\prod_{i=1}^{r-1} m_{i} n_{i}\right)^{1 / 6}}\right) \\
\quad \times \prod_{i=1}^{r-1}\left(\frac{t_{i+1} \wedge t_{i+1}^{\prime}-t_{i} \vee t_{i}^{\prime}}{\sqrt{\left(t_{i+1}-t_{i}\right)\left(t_{i+1}^{\prime}-t_{i}^{\prime}\right)}}\right)^{m_{i}+n_{i}},
\end{aligned}
$$

where $a \wedge b=\min (a, b)$ and $a \vee b=\max (a, b)$.

Proof. We prove (ii) first. Since $u_{i}$ is positive, $\mid\left\langle u_{i}, f g\right\rangle \leqslant\left\langle u_{i}, f\right\rangle \cdot\|g\|_{\infty}$ for all $f, g \in \mathscr{S}\left(R^{2}\right)$ and $f \geqslant 0$. Thus

$$
\begin{gathered}
\mid\left\langle u_{i}\left(x_{1}, x_{2}\right), H_{m_{i}}\left(\frac{x_{1}}{\sqrt{2\left(t_{i+1}-t_{i}\right)}}\right) H_{n_{i}}\left(\frac{x_{2}}{\sqrt{2\left(t_{1+1}-t_{i}\right)}}\right) \exp \left(-\frac{x_{1}^{2}+x_{2}^{2}}{2\left(t_{i+1}-t_{i}\right)}\right\rangle\right| \\
\leqslant\left(\left\langle u_{i}(x), \exp \left(-\frac{|x|^{2}}{4\left(t_{i+1}-t_{i}\right)}\right)\right\rangle_{x=\left(x_{1}, x_{2}\right)}\right)
\end{gathered}
$$




$$
\times \sup _{\substack{t>0 \\ x_{1}, x_{2} \in R}}\left|\exp \left(-\frac{x_{1}^{2}+x_{2}^{2}}{4 t}\right) H_{m_{i}}\left(\frac{x_{1}}{\sqrt{2 t}}\right) H_{n_{i}}\left(\frac{x_{2}}{\sqrt{2 t}}\right)\right| .
$$

The first term in the above product is less than a constant. This is because $t_{i+1}-t_{i} \geqslant a_{i+1}-b_{i} \geqslant \theta>0$ and the mapping $t \in\left[T_{0}, T_{1}\right] \rightarrow\left\langle u_{i}(\cdot)\right.$, $\left.\exp \left(-\left.|\cdot|\right|^{2} / 4 t\right)\right\rangle$ is continuous, hence is bounded, on each $\left[T_{0}, T_{1}\right], 0<T_{0}<$ $T_{1}<\infty$. The second term is $O\left(\left(m_{i} n_{i} 2^{m_{i}} 2^{n_{i}}\right)^{1 / 2}\left(m_{i} n_{i}\right)^{-1 / 12}\right)$, according to Szegö $[21,(8.91 .10)]$. Thus, the expectation in (ii) is not greater than a constant multiple of

$$
\left(\prod_{i=1}^{r-1} \frac{1}{m_{i} ! n_{i} ! 2^{m_{i}} 2^{n_{i}} \sqrt[6]{m_{i} n_{i}}}\right) E\left(\prod_{i=1}^{r-1} H_{m_{i}}\left(\frac{X_{i}}{\sqrt{2}}\right) H_{m_{i}}\left(\frac{X_{i}^{\prime}}{\sqrt{2}}\right) H_{n_{i}}\left(\frac{Y_{i}}{\sqrt{2}}\right) H_{n_{i}}\left(\frac{Y_{i}^{\prime}}{\sqrt{2}}\right)\right)
$$

where

$$
\begin{aligned}
& X_{i}(\omega)=\frac{\left\langle\omega, 1_{\left[t_{i}, t_{i+1}\right]}\right\rangle}{\sqrt{t_{i+1}-t_{i}}}, \quad X_{i}^{\prime}(\omega)=\frac{\left\langle\omega, 1_{\left[t_{\left.i^{\prime}, t_{i+1}\right]}\right.}\right\rangle}{\sqrt{\bar{t}_{i+1}^{\prime}-t_{1}^{\prime}}} \\
& Y_{i}(\omega)=\frac{\left\langle\omega, 1_{\left[-t_{i+1},-t_{i}\right]}\right\rangle}{\sqrt{\left(t_{i+1}-t_{i}\right)}}, \quad Y_{i}^{\prime}(\omega)=\frac{\left\langle\omega, 1_{\left[-t^{\prime} i+1, t_{i}^{\prime}\right]}\right\rangle}{\sqrt{\left(t_{1+1}^{\prime}-t_{i}^{\prime}\right)}} .
\end{aligned}
$$

Since $X_{i}$ and $X_{i}^{\prime}$ are independent of $Y_{i}$ and $Y_{i}^{\prime}$, the expectation in (3.2) is equal to the product of (3.3) and (3.4) below, and (ii) will follow from these two displays once they have been proved.

$$
\begin{aligned}
& E \prod_{i=1}^{r-1} H_{m_{i}}\left(\frac{X_{i}}{\sqrt{2}}\right) H_{m_{i}}\left(\frac{X_{i}^{\prime}}{\sqrt{2}}\right) \\
& \quad=\left(\frac{\prod_{i=1}^{r-1}\left(m_{i} !\right)^{2}}{\left(\sum_{i=1}^{r-1} m_{i}\right) !} 2^{\Sigma_{i=1}^{r-1} m_{i}}\right) \prod_{i=1}^{r-1}\left(\frac{t_{i+1} \wedge t_{i+1}^{\prime}-t_{i} \vee t_{i}^{\prime}}{\sqrt{\left(t_{i+1}-t_{i}\right)\left(t_{i+1}^{\prime}-t_{i}^{\prime}\right)}}\right)^{m_{2}}, \\
& E \prod_{i=1}^{r-1} H_{n_{i}}\left(\frac{Y_{i}}{\sqrt{2}}\right) H_{n_{i}}\left(\frac{Y_{i}^{\prime}}{\sqrt{2}}\right) \\
& =\left(\frac{\prod_{i=1}^{r-1}\left(n_{i} !\right)^{2}}{\left(\sum_{i=1}^{r-1} n_{i}\right) !} 2^{\Sigma_{i=1}^{r-1} n_{i}}\right) \prod_{i=1}^{r-1}\left(\frac{t_{i+1} \wedge t_{i+1}^{\prime}-t_{i} \vee t_{i}^{\prime}}{\sqrt{\left(t_{i+1}-t_{i}\right)\left(t_{i+1}^{\prime}-t_{i}^{\prime}\right)}}\right)^{n_{i}} .
\end{aligned}
$$

We prove (3.3), and (3.4) is proved in the same way. Each $X_{i}$ and $X_{i}^{\prime}$ is $N(0,1)$ distributed. In case $r=2$, we apply the ready formula in Hida $[7$, p. 139] and the covariance

$$
E\left(X_{i} X_{i}^{\prime}\right)=\frac{t_{i+1} \wedge t_{i+1}^{\prime}-t_{i} \vee t_{i}^{\prime}}{\sqrt{\left(t_{i+1}-t_{i}\right)\left(t_{i+1}^{\prime}-i_{i}^{\prime}\right)}}
$$

In case $r>2$, we appeal to the diagram formula listed in (for example) Major [14, p. 51]. Note that, in our case, the summation in the diagram 
formula is reduced to only one term, namely the right-handed side of (3.3). This is due to the covariances $E\left(X_{i} X_{i}^{\prime}\right)$ and

$$
\begin{aligned}
& E\left(X_{i} X_{i+1}^{\prime}\right)=\frac{\left(t_{i+1}-t_{i+1}^{\prime}\right)^{+}}{\sqrt{\left(t_{i+1}-t_{i}\right)\left(t_{i+2}^{\prime}-t_{i+1}^{\prime}\right)}}, \quad a^{+}=\max (a, 0), \\
& E\left(X_{i+1} X_{i}^{\prime}\right)=\frac{\left(t_{i+1}^{\prime}-t_{i+1}\right)^{+}}{\sqrt{\left(t_{i+2}-t_{i+1}\right)\left(t_{i+1}^{\prime}-t_{i}^{\prime}\right)}},
\end{aligned}
$$

and that all other $X_{i}$ and $X_{i}^{\prime}$ have zero correlations. In the above, we rely on the relation $t_{i+1}, t_{i+1}^{\prime}>t_{i}, t_{i}^{\prime}$.

The diagram formula also asserts the validity of (i), thus, the proof of Lemma 3.1 is completed.

Now, we prove Proposition 3.1. By (2.2), the series expansion of (3.1) is

$$
\frac{1}{(2 \pi)^{r-1}} \sum_{\substack{\left(m_{i}, n_{i}\right) \\ i=1, \ldots, r-1}} \int_{a_{r}}^{b_{r}} \cdots \int_{a_{1}}^{b_{1}} \frac{\prod_{i=1}^{r-1} \phi_{i}\left(m_{i}, n_{i}, t_{i}, t_{i+1}\right)}{\prod_{i=1}^{r-1}\left(t_{i+1}-t_{i}\right)} d t_{1} \cdots d t_{r} .
$$

Let the integral in the above summation be denoted by $\psi\left(m_{1}, n_{1}, \cdots, m_{r-1}\right.$, $\left.n_{r-1}\right)$. By Lemma 3.1 (i), $\psi\left(m_{i}, n_{i}\right)$ 's are orthogonal in $\left(L^{2}\right)$. By Lemma 3.1 (ii), the square of the $\left(L^{2}\right)$ norm of $\psi\left(m_{j}, n_{j}\right)$ is not greater than a constant multiple of

$$
\begin{aligned}
& \left(\frac{\prod_{i=1}^{r-1} m_{i} ! n_{i} !}{\left(\sum_{i=1}^{r-1} m_{i}\right) !\left(\sum_{i=1}^{r-1} n_{i}\right) !\left(\prod_{i-1}^{r-1} m_{i} n_{i}\right)^{1 / 6}}\right) \int_{t_{r}, t_{r}^{\prime}=a_{r}}^{b_{r}} \cdots \int_{t_{1}, t_{r}^{\prime}=a_{1}}^{b_{1}} \\
& \quad \times \prod_{i=1}^{r-1} \frac{\left(t_{i+1} \wedge t_{i+1}^{\prime}-t_{i} \vee t_{i}^{\prime}\right)^{m_{i}+n_{i}}}{\left(\left(t_{i+1}-t_{i}\right)\left(t_{i+1}^{\prime}-t_{i}^{\prime}\right)\right)^{\left(m_{i}+n_{i}\right) / 2+1}} d t_{1} \cdots d t_{t} d t_{1}^{\prime} \cdots d t_{r}^{\prime} .
\end{aligned}
$$

(i) $r=2$. That (3.5) is reduced to

$$
\frac{1}{\sqrt[6]{m n}} \int_{t_{2}, t_{2}^{\prime}} \int_{t_{1}, t_{1}^{\prime}} \frac{\left(t_{2} \wedge t_{2}^{\prime}-t_{1} \vee t_{1}^{\prime}\right)^{m+n}}{\left(\left(t_{2}-t_{1}\right)\left(t_{2}^{\prime}-t_{1}^{\prime}\right)\right)^{m+n) / 2+1}} d t_{1} d t_{2} d t_{1}^{\prime} d t_{2}^{\prime}
$$

The integration domain of $t_{1}, t_{1}^{\prime}, t_{2}, t_{2}^{\prime}$ is split into four parts. On $t_{1} \leqslant t_{1}^{\prime}$ $<t_{2} \leqslant t_{2}^{\prime}$, we integrate in the order of $t_{2}^{\prime}, t_{1}^{\prime}, t_{2}, t_{1}$ and find that the resultant is $\leqslant$ const. $/(m+n)^{2}$. On $t_{1} \leqslant t_{1}^{\prime}<t_{2}^{\prime} \leqslant t_{2}$, we integrate in the order of $t_{1}^{\prime}, t_{2}^{\prime}, t_{2}, t_{1}$ and find that the same dominating factor $1 /(m+n)^{2}$ appears. The other two parts are treated similarly. We conclude that (3.6) is dominated by a constant multiple of

$$
\frac{1}{(m+n)^{2} \sqrt[6]{m n}} \leqslant \frac{1}{4(m n)^{7 / 6}} .
$$


Therefore, the series expansion of (3.7), for $r=2$, converges in $\left(L^{2}\right)$, and thus (3.1), for $r=2$, is in $\left(L^{2}\right)$.

(ii) $r>2$. The integration domain of $t_{1}, t_{1}^{\prime}, \cdots, t_{r}, t_{r}^{\prime}$ is split into $2^{r}$ parts. On $t_{1} \leqslant t_{1}^{\prime}<t_{2} \leqslant t_{2}^{\prime}<\cdots<t_{r} \leqslant t_{r}^{\prime}$, we integrate in the order of $t_{r}^{\prime}$, $t_{r-1}^{\prime}, \cdots, t_{1}^{\prime}$ and in the meantime drop the terms involving $\left(t_{i}-t_{i-1}^{\prime}\right) /\left(t_{i}-t_{i-1}\right)$, the resultant $\leqslant$ const. $/\left(m_{r-1}+n_{r-1}\right) \cdots\left(m_{1}+n_{1}\right)$ (Compare with (i); we have no $\left(m_{i}+n_{i}\right)^{2}$ here). We treat all other parts similarly, and conclude that (3.5) is dominated by a constant multiple of

$$
\frac{\prod_{i=1}^{r-1} m_{i} ! n_{i} !}{\left(\sum_{i=1}^{r-1} m_{i}\right) !\left(\sum_{i=1}^{r-1} n_{i}\right) ! \prod_{i=1}^{r-1}\left(m_{i}+n_{i}\right) \sqrt[6]{m_{i} n_{i}}} .
$$

Using $m_{i}+n_{i} \geqslant 2 \sqrt{m_{i} n_{i}}$ and Stirling formula, we see that the above is not greater than a constant multiple of

$$
\left(\frac{\prod_{i=1}^{r-1} m_{i}^{m_{i}+1} n_{i}^{n_{i}+1}}{\left(\sum_{i=1}^{r-1} m_{i}\right)^{1 / 2+\Sigma m_{i}}\left(\sum_{i=1}^{r-1} n_{i}\right)^{1 / 2+\Sigma n_{i}}}\right) \frac{1}{\left(\prod_{i=1}^{r-1} m_{i} n_{i}\right)^{7 / 6}} .
$$

Since the quantity in the parenthesis above is $\leqslant 1$, we have shown again that the series expansion of (3.1) converges in $\left(L^{2}\right)$. This completes the proof.

Remark. The above proof depends on the off-diagonal assumption $a_{i}>b_{i-1}$. In the diagonal case $\left\{t_{1} \leqslant t_{2} \leqslant \cdots \leqslant T\right\}$, we even cannot assert that (3.1) is in $\left(L^{2}\right)^{-}$, since the limiting case $\lim _{t \downarrow s} u(B(t)-B(s))$ is in general unknown. Thus, it seems challenging to "renormalize" (3.1) in this case.

Let $f(x)$ be a measurable function on $R^{2}$, which is locally squareintegrable and is slowly increasing as $|x| \rightarrow \infty$. That $\log |x|$ is such a function. Then, for $0 \leqslant s \leqslant t$,

$$
E f^{2}(B(t)-B(s))=\frac{1}{2 \pi(t-s)} \int_{R^{2}} f^{2}(x) \exp \left(-\frac{x^{2}}{2(t-s)}\right) d x<\infty .
$$

Proposition 3.2. Assume that $a_{1}<b_{1}<a_{2}<b_{2} \cdots$, $u_{i}$ are given in Proposition 3.1 and $f(x)$ is the function mentioned above. Then, for each $t \in\left[a_{r+1}, b_{r+1}\right]$,

$$
\int_{a_{r}}^{b_{r}} \cdots \int_{a_{1}}^{b_{1}} f\left(B(t)-B\left(t_{r}\right)\right) \prod_{i=1}^{r-1} u_{i}\left(B\left(t_{i+1}\right)-B\left(t_{i}\right)\right) d t_{1} \cdots d t_{r}
$$

is in $\left(L^{2}\right)$. 
Proof. As in the proof of Proposition 3.1, the series expansion of (3.8) is

$$
\frac{1}{(2 \pi)^{r}} \sum_{\substack{\left(m_{i}, n_{i}\right) \\ i=1, \cdots, r}} \int_{a_{r}}^{b_{r}} \cdots \int_{a_{1}}^{b_{1}} \frac{\prod_{i=1}^{r} \phi_{i}\left(m_{i}, n_{i}, t_{i}, t_{i+1}\right)}{\prod_{i=1}^{r}\left(t_{i+1}-t_{i}\right)} d t_{1} \cdots d t_{r},
$$

where $t_{r+1}=t$ and $\phi_{r}\left(m_{r}, n_{r}, t_{r}, t_{r+1}\right)$ is the term corresponding to $f\left(B\left(t_{r+1}\right.\right.$ $\left.-B\left(t_{r}\right)\right)$. To estimate the squares of $\left(L^{2}\right)$ norms of the above summunds and their sum, we note that

$$
\frac{1}{2 \pi\left(t_{r+1}-t_{r}\right)} \sum_{m_{r}, n_{r}} \phi_{r}\left(m_{r}, n_{r}, t_{r}, t_{r+1}\right)
$$

is the series expansion of $f\left(B\left(t_{r+1}\right)-B\left(t_{r}\right)\right)$ in $\left(L^{2}\right)$. Thus,

$$
\begin{array}{rl}
\sum_{m_{r}, n_{r}} & E \prod_{i=1}^{r} \frac{\phi_{i}\left(m_{i}, n_{i}, t_{i}, t_{i+1}\right) \phi_{i}\left(m_{i}, n_{i}, t_{i}^{\prime}, t_{i+1}^{\prime}\right)}{\left(t_{i+1}-t_{i}\right)\left(t_{i+1}^{\prime}-t_{i}\right)}, t_{r+1}=t_{r+1}^{\prime}=t \\
& =\left[E \prod_{i=1}^{r-1} \cdots\right] \cdot(4 \pi)^{2} E\left[f\left(B(t)-B\left(t_{r}\right)\right) f\left(B(t)-B\left(t_{r}^{\prime}\right)\right)\right] \\
& \leqslant\left[E \prod_{i=1}^{r-1} \cdots\right]\left[\frac{2 \pi}{\left(t-b_{r}\right)} \int_{R^{2}} f^{2}(x) \exp \left(-\frac{x^{2}}{\left(t-a_{r}\right)}\right) d x\right]
\end{array}
$$

Therefore, the arguments in the proof of Proposition 3.1 show that (3.9) converges in $\left(L^{2}\right)$.

\section{\$4. A Tanaka type formula and the proof of Theorem 1.1}

Let $0<a_{1}<b_{1}<a_{2}<b_{2}<\cdots<a_{r+1}<b_{r+1}$ and $u_{1}, \cdots, u_{r-1} \in \mathscr{S}^{*}\left(R^{2}\right)$ be positive, we shall prove

THEOREM 4.1. Let $f(x), x=\left(x_{1}, x_{2}\right) \in R^{2}$, be a measurable function such that $f(x)$ itself and its distributional derivatives $\partial f / \partial x_{1}, \partial f / \partial x_{2}$ are all locally square-integrable and slowly increasing as $|x| \rightarrow \infty$. Let $\Delta f$ be the distributional Liplacian of $f$. Assume also that $\Delta f \in \mathscr{S}^{*}\left(R^{2}\right)$ is positive. Then the following equality holds in $\left(L^{2}\right)$.

$$
\begin{aligned}
\int_{a_{r}}^{b_{r}} \cdots \int_{a_{1}}^{b_{1}}\left\{f\left(B\left(b_{r+1}\right)-B\left(t_{r}\right)\right) \prod_{i=1}^{r-1} u_{i}\left(B\left(t_{i+1}\right)-B\left(t_{i}\right)\right)\right\} d t_{1} \cdots d t_{r} \\
-\int_{a_{r}}^{b_{r}} \cdots \int_{a_{1}}^{b_{1}}\left\{f\left(B\left(a_{r+1}\right)-B\left(t_{r}\right)\right) \prod_{i=1}^{r-1} u_{i}\left(B\left(t_{i+1}\right)-B\left(t_{i}\right)\right)\right\} d t_{1} \cdots d t_{r} \\
=\sum_{j=1}^{2} \int_{a_{r+1}}^{b_{r+1}}\left(\int _ { a _ { r } } ^ { b _ { r } } \cdots \int _ { a _ { 1 } } ^ { b _ { 1 } } \left\{\frac{\partial f}{\partial x_{j}}\left(B\left(t_{r+1}\right)-B\left(t_{r}\right)\right)\right.\right. \\
\left.\left.\times \prod_{i=1}^{r-1} u_{i}\left(B\left(t_{i+1}\right)-B\left(t_{i}\right)\right)\right\} d t_{1} \cdots d t_{r}\right) d B_{j}\left(t_{r+1}\right)
\end{aligned}
$$




$$
\begin{aligned}
+\frac{1}{2} \int_{a_{r+1}}^{b_{r+1}} \cdots \int_{a_{1}}^{b_{1}}\{(\Delta f) & \left(B\left(t_{r+1}\right)-B\left(t_{r}\right)\right) \\
& \left.\times \prod_{i=1}^{r-1} u_{i}\left(B\left(t_{r+1}\right)-B\left(t_{i}\right)\right)\right\} d t_{1} \cdots d t_{r+1} .
\end{aligned}
$$

In the above, the first term on the right-handed side is an Ito's integral.

Proof. For each $t_{1}, \cdots, t_{r}$, fixed, we have the following Kubo's formula in $\left(L^{2}\right)^{-}$

$$
\begin{aligned}
\frac{d}{d t_{r+1}}\{ & \left.f\left(B\left(t_{r+1}\right)-B\left(t_{r}\right)\right) \prod_{i=1}^{r-1} u_{i}\left(B\left(t_{i+1}\right)-B\left(t_{i}\right)\right)\right\} \\
= & \partial_{t_{r+1}}^{*}\left\{(\nabla f)\left(B\left(t_{r+1}\right)-B\left(t_{r}\right)\right) \prod_{i=1}^{r-1} u_{i}\left(B\left(t_{i+1}\right)-B\left(t_{i}\right)\right)\right\} \\
& +\frac{1}{2}\left\{(\Delta f)\left(B\left(t_{r+1}\right)-B\left(t_{r}\right)\right) \prod_{i=1}^{r-1} u_{i}\left(B\left(t_{i+1}\right)-B\left(t_{i}\right)\right)\right\} .
\end{aligned}
$$

The above equality can be proved by calculating the $\mathscr{S}$-transform of each term, as Kubo did. Now, integrating in $t_{1}, \cdots, t_{r+1}$, we have the following equality in $\left(L^{2}\right)^{-}$:

$$
\begin{gathered}
\int_{a_{r}}^{b_{r}} \cdots \int_{a_{1}}^{b_{1}}\left\{f\left(B\left(b_{r+1}\right)-B\left(t_{r}\right)\right) \prod_{i=1}^{r-1} u_{i}\left(B\left(t_{i+1}\right)-B\left(t_{i}\right)\right)\right\} d t_{1} \cdots d t_{r} \\
-\int_{a_{r}}^{b_{r}} \cdots \int_{a_{1}}^{b_{1}}\left\{f\left(B\left(a_{r+1}\right)-B\left(t_{r}\right)\right) \prod_{i=1}^{r-1} u_{i}\left(B\left(t_{i+1}\right)-B\left(t_{i}\right)\right)\right\} d t_{1} \cdots d t_{r} \\
=\int_{a_{r+1}}^{b_{r+1}} \cdots \int_{a_{1}}^{b_{1}}\left\{\partial_{t_{r+1}}^{*}(\nabla f)\left(B\left(t_{r+1}\right)-B\left(t_{r}\right)\right)\right. \\
\left.\quad \times \prod_{i=1}^{r-1} u_{i}\left(B\left(t_{i+1}\right)-B\left(t_{i}\right)\right)\right\} d t_{1} \cdots d t_{r} d t_{r+1} \\
+\frac{1}{2} \int_{a_{r+1}}^{b_{r+1}} \cdots \int_{a_{1}}^{b_{1}}\left\{(\Delta f)\left(B\left(t_{r+1}\right)-B\left(t_{r}\right)\right)\right. \\
\left.\times \prod_{i=1}^{r-1} u_{i}\left(B\left(t_{i+1}\right)-B\left(t_{i}\right)\right)\right\} d t_{1} \cdots d t_{r} d t_{r+1} .
\end{gathered}
$$

Propositions 3.1 and 3.2 assert respectively that the last term and the left-handed side of (4.2) are in $\left(L^{2}\right)$. Therefore, the first term on the right-handed side is also in $\left(L^{2}\right)$ and (4.2) becomes an a.s. eauality. By Proposition 3.2,

$$
\begin{aligned}
t_{r+1} & \in\left[a_{r+1}, b_{r+1}\right] \\
& \rightarrow \int_{a_{r}}^{b_{r}} \cdots \int_{a_{1}}^{b_{1}}\left\{\frac{\partial f}{\partial x_{j}}\left(B\left(t_{r+1}\right)-B\left(t_{r}\right)\right) \prod_{i=1}^{r-1} u_{i}\left(B\left(t_{i+1}\right)-B\left(t_{i}\right)\right)\right\} d t_{1} \cdots d t_{r}
\end{aligned}
$$

is an nonanticipating $\left(L^{2}\right)$ process. Thus, according to Kubo [11, Theorem 
2.1] or Hida [8, the concluding statement], the two first terms on the right-handed sides of (4.1) and (4.2) must be equal a.s., and (4.1) has been proved.

To derive Theorem 1.1, we need the following lemma.

LEMma 4.2. The following equalities hold in $\left(L^{2}\right)$.

$$
\begin{aligned}
& \int_{a_{r}}^{b_{r}} \cdots \int_{a_{1}}^{b_{1}} \prod_{i=1}^{r-1} \delta_{x_{i}}\left(B\left(t_{i+1}\right)-B\left(t_{i}\right)\right) d t_{1} \cdots d t_{r} \\
& =\alpha_{r}\left(x, a_{1}, b_{1}, \cdots, a_{r}, b_{r}\right), \quad x=\left(x_{1}, \cdots, x_{r-1}\right) \in R^{2(r-1)} . \\
& \text { (ii) } \quad \int_{a_{r}}^{b_{r}} \cdots \int_{a_{1}}^{b_{1}} f\left(B(t)-B\left(t_{r}\right)\right) \prod_{i=1}^{r-1} \delta_{x_{i}}\left(B\left(t_{i+1}\right)-B\left(t_{i}\right)\right) d t_{1} \cdots d t_{r} \\
& =\int_{a_{r}}^{b_{r}} f\left(B(t)-B\left(t_{r}\right)\right) \alpha_{r}\left(x, a_{1}, b_{1}, \cdots, a_{r}, d t_{r}\right),
\end{aligned}
$$

where $t \in\left[a_{r+1}, b_{r+1}\right]$ and $f(y)$ is the function in Proposition 3.2.

Proof. By Propositions 3.1 and 3.2, the left-handed sides of (i) and (ii) are in $\left(L^{2}\right)$. Set

$$
\begin{aligned}
& \alpha_{r}^{\varepsilon}\left(x, a_{1}, \cdots, b_{r}\right)=\int_{a_{r}}^{b_{r}} \cdots \int_{a_{1}}^{b_{1}} \prod_{i=1}^{r-1} p_{\varepsilon}\left(B\left(t_{i+1}\right)-B\left(t_{i}\right)-x_{i}\right) d t_{1} \cdots d t_{r}, \\
& \beta_{r}^{\varepsilon}\left(x, a_{1}, \cdots, b_{r}\right) \\
& \quad=\int_{a_{r}}^{b_{r}} \cdots \int_{a_{1}}^{b_{1}} f\left(B(t)-B\left(t_{r}\right)\right) \prod_{i=1}^{r-1} p_{\varepsilon}\left(B\left(t_{i+1}\right)-B\left(t_{i}\right)-x_{i}\right) d t_{1} \cdots d t_{r},
\end{aligned}
$$

where $p_{\varepsilon}(y)$ is the standard planar $N(0, \varepsilon)$ density function. On one hand, by computing the $\mathscr{S}$-transforms we see that $\alpha_{r}^{\varepsilon}$ and $\beta_{r}^{s}$ converge respectively in $\left(L^{2}\right)^{-}$to the left-handed sides of (i) and (ii). On the other hand, by Rosen [17, Lemma 1 and p. 135] we see that $\alpha_{r}^{\varepsilon}$ converges in $\left(L^{2}\right)$ to the right-handed side of (i) and $\beta_{r}^{s}$ converges pathwise to the right-handed side of (ii). This proves the assertion.

We cannot derive (1.2) directly from Theorem 4.1, since $\left(x_{1}, x_{2}\right) \rightarrow$ $x_{i} /\left(x_{1}^{2}+x_{2}^{2}\right)$ is merely in $L_{\text {loc }}^{1}\left(R^{2}\right)$ not in $L_{\text {loc }}^{2}\left(R^{2}\right)$. However, we can prove (1.2) as follows. Let $f_{\varepsilon}(y)=(1 / 2 \pi) \log \left(|y|^{2}+\varepsilon^{2}\right)^{1 / 2}, 0<\varepsilon \leqslant 1$ and $y \in R^{2}$. For each $x=\left(x_{1}, \cdots, x_{r+1}\right) \in R^{2(r-1)}$ and $x_{r} \in R^{2}$, by Theorem 4.1 and Lemma 4.2 the following equality holds in $\left(L^{2}\right)$.

$$
\begin{aligned}
\int_{a_{r}}^{b_{r}} f_{\varepsilon}\left(B\left(b_{r+1}\right)-B\left(t_{r}\right)-x_{r}\right) \alpha_{r}\left(x, a_{1}, \cdots, a_{r}, d t_{r}\right) \\
\quad-\int_{a_{r}}^{b_{r}} f_{\varepsilon}\left(B\left(a_{r+1}\right)-B\left(t_{r}\right)-x_{r}\right) \alpha_{r}\left(x, a_{1}, \cdots, a_{r}, d t_{r}\right)
\end{aligned}
$$




$$
\begin{aligned}
& =\int_{a_{r+1}}^{b_{r+1}}\left(\int_{a_{r}}^{b_{r}}\left(\nabla f_{\varepsilon}\right)\left(B\left(t_{r+1}\right)-B\left(t_{r}\right)-x_{r}\right) \alpha_{r}\left(x, a_{1}, \cdots, a_{r}, d t_{r}\right)\right) \cdot d B\left(t_{r+1}\right) \\
& \quad+\frac{1}{2} \int_{y \in R^{2}}\left(\Delta f_{\varepsilon}\right)\left(y-x_{r}\right) \alpha_{r+1}\left((x, y), a_{1}, \cdots, b_{r+1}\right) d y,
\end{aligned}
$$

where the last term is due to the definition of $\alpha_{r+1}$ and the fact that $\Delta f_{\varepsilon}$ is a positive continuous function. Since

$$
\int_{y \in R^{2}}\left(f_{\varepsilon}\left(y-x_{r}\right)-\frac{1}{2 \pi} \log \left|y-x_{r}\right|\right)^{2} \exp \left(-\frac{|y|^{2}}{2 s}\right) d y \rightarrow 0 \quad \text { for all } s>0,
$$

by Lemma 4.2 and (3.10) in the proof of Proposition 3.2 the left-handed side of (4.3) converges in $\left(L^{2}\right)$ to that of (1.2) as $\varepsilon \downarrow 0$. Since $\left(\Delta f_{\varepsilon}\right)(y)=$ $\left(\varepsilon^{2} / \pi\right)\left(1 /\left(|x|^{2}+\varepsilon^{2}\right), 0<\varepsilon \leqslant 1\right.$, is an approximation to the identity on $R^{2}$, by Rosen [17, Lemma 1 and its proof], the last term of (4.3) converges in $\left(L^{2}\right)$ to $\alpha_{r+1}\left(\left(x, x_{r}\right), a_{1}, \cdots, b_{r+1}\right)$ as $\varepsilon \downarrow 0$. Therefore, the stochastic integral term of (4.3) must also converge in $\left(L^{2}\right)$. By the isometry property of stochastic integrals, the limit must be of the form

$$
\int_{a_{r+1}}^{b_{r+1}} \phi_{r}\left(t_{r+1}, \omega\right) \cdot d B\left(t_{r+1}, \omega\right)
$$

where $\phi_{r}\left(t_{r+1}, \omega\right)$ is the $L^{2}\left(d t_{r+1} \times d P\right)$ limit of

$$
\int_{a_{r}}^{b_{r}} \frac{B\left(t_{r+1}\right)-B\left(t_{r}\right)-x_{r}}{(2 \pi)\left(\left|B\left(t_{r+1}\right)-B\left(t_{r}\right)-x_{r}\right|^{2}+\varepsilon^{2}\right)} \alpha_{r}\left(x, a_{1}, \cdots, a_{r}, d t_{r}\right)
$$

since $\left(\nabla f_{\varepsilon}\right)(y)=y /(2 \pi)\left(|y|^{2}+\varepsilon^{2}\right)$. Note that $1 /\left(|y|^{2}+\varepsilon^{2}\right) \uparrow 1 /|y|^{2}$. Thus, by the monotone convergence, for a.e. $t_{r+1}$

$$
\begin{gathered}
\int_{a_{r}}^{b_{r}} \frac{B\left(t_{r+1}, \omega\right)-B\left(t_{r}, \omega\right)-x_{r}}{(2 \pi)\left|B\left(t_{r+1}, \omega\right)-B\left(t_{r}, \omega\right)-x_{r}\right|^{2}} \alpha_{r}\left(x, a_{1}, \cdots, a_{r}, d t_{r}, \omega\right) \\
\quad=\phi_{r}\left(t_{r+1}, \omega\right), \text { which is of finite value for a.s. } \omega .
\end{gathered}
$$

Hence, we have proved that the integrand of the stochastic integral in (1.2) is well-defined and that (1.2) holds in $\left(L^{2}\right)$.

\section{§5. An explicit form of $\tilde{\alpha}_{r}(x, T)$}

We have mentioned in $\S 1$ that, in the diagonal case, we intend to substract off certain "infinite part" from $\alpha_{r}(x, T)$ so that the remainder $\tilde{\alpha}_{r}(x, T)$ will have a jointly continuous extension to all $(x, T) \in R^{2(r-1)} \times R_{+}$. In this section, we shall discuss how (1.2) suggests an explicit form of such a "cut-off". We shall show that the explicit forms in Yor [23, $r=2]$ 
and Rosen-Yor [20, $r=3$ ] can be extended to general $r$. Recall that

$$
\tilde{\alpha}_{2}(x, T)=\alpha_{2}(x, T)-\frac{T}{\pi} \log \frac{1}{|x|}, \quad x \in R^{2} \text { and } x \neq 0 .
$$

Renormalization of $\alpha_{r}$. For each $r \geqslant 3$, suppose that $\tilde{\alpha}_{2}, \cdots, \tilde{\alpha}_{r-1}$ have been constructed. Set

$$
N(x)=\frac{1}{\pi} \log \frac{1}{|x|}, \quad x \neq R^{2} \text { and } x \neq 0 .
$$

Then, we can construct $\tilde{\alpha}_{r}$ as follows.

$$
\begin{aligned}
\tilde{\alpha}_{r}\left(x_{1}, \cdots, x_{r-1}, T\right)=\alpha_{r}\left(x_{1}, \cdots, x_{r-1}, T\right) \\
-\sum_{i=1}^{r} N\left(x_{i}\right) \tilde{\alpha}_{r-1}(\overbrace{x_{1}, \cdots, x_{r-1}}^{i} T) \\
\quad-\sum_{i, j=1}^{r} N\left(x_{i}\right) N\left(x_{j}\right) \tilde{\alpha}_{r-2}(\overbrace{x_{1}, \cdots, x_{r-1}}^{i, j} T) \\
\quad-\sum_{i, j, k=1}^{r} N\left(x_{i}\right) N\left(x_{j}\right) N\left(x_{k}\right) \tilde{\alpha}_{r-3}(\overbrace{x_{1}, \cdots, x_{r-1}}^{i, j, k} T) \\
\quad-N\left(x_{1}\right) \cdots N\left(x_{r-1}\right) T ;
\end{aligned}
$$

in the above, $\overbrace{x_{1}, \cdots, x_{r-1}}^{i}$ indicates that the $i$-th one has been deleted. etc.

Thus, in case $r=3$, we have

$$
\begin{aligned}
& \tilde{\alpha}_{3}\left(x_{1}, x_{2}, T\right)=\alpha_{3}\left(x_{1}, x_{2}, T\right)-\frac{1}{\pi} \log \frac{1}{\left|x_{1}\right|} \tilde{\alpha}_{2}\left(x_{2}, T\right) \\
& -\frac{1}{\pi} \log \frac{1}{\left|x_{2}\right|} \tilde{\alpha}_{2}\left(x_{1}, T\right)-\frac{T}{\pi^{2}} \log \frac{1}{\left|x_{1}\right|} \log \frac{1}{\left|x_{2}\right|},
\end{aligned}
$$

which is consistant with the one obtained by Rosen-Yor [20]. We show in the below how to obtain $\tilde{\alpha}_{4}$ from $\tilde{\alpha}_{2}$ and $\tilde{\alpha}_{3}$ by using Theorem 1.1 (it is in the same way to obtain $\tilde{\alpha}_{3}$ from $\tilde{\alpha}_{2}$ ). First, we may rewrite (1.2) as follows. For each $T>0$, each $x_{1}, x_{2}, x_{3} \in R^{2}$ and $x_{i} \neq 0$, with probability one

$$
\begin{gathered}
\int_{0}^{T} \log \left|B(T)-B(t)-x_{3}\right| \alpha_{3}\left(x_{1}, x_{2}, d t\right) \\
\quad-\int_{0}^{T} \log \left|B(t)-B(t)-x_{3}\right| \alpha_{3}\left(x_{1}, x_{2}, d t\right) \\
=\int_{0}^{T} \int_{0}^{u} \frac{B(u)-B(t)-x_{3}}{\left|B(u)-B(t)-x_{3}\right|^{2}} \alpha_{3}\left(x_{1}, x_{2}, d t\right) \cdot d B(u)+\pi \alpha_{4}\left(x_{1}, x_{2}, x_{3}, T\right) .
\end{gathered}
$$


The proof of the above equality is parallel to that of (1.2), with the simple observation that $\lim _{t \downarrow 0}(1 / t) \exp \left(-a^{2} / 2 t\right)=0$ for all $a \neq 0$. Now, we write $\tilde{\alpha}_{3}\left(x_{1}, x_{2}, d t\right)=\alpha_{3}\left(x_{1}, x_{2}, d t\right)-N_{3}\left(x_{1}, x_{2}, d t\right)$, where $N_{3}$ denotes the "infinite part". Thus, we have, by (5.4),

$$
\begin{gathered}
\int_{0}^{T} \log \left|B(T)-B(t)-x_{3}\right| \tilde{\alpha}_{3}\left(x_{1}, x_{2}, d t\right) \\
\quad-\int_{0}^{T} \int_{0}^{u} \frac{B(u)-B(t)-x_{3}}{\left|B(u)-B(t)-x_{3}\right|^{2}} \tilde{\alpha}_{3}\left(x_{1}, x_{2}, d t\right) \cdot d B(u) \\
=\pi \alpha_{4}\left(x_{1}, x_{2}, x_{3}, T\right)-\log \frac{1}{\left|x_{3}\right|} \tilde{\alpha}_{3}\left(x_{1}, x_{2}, T\right)-\log \frac{1}{\left|x_{3}\right|} N_{3}\left(x_{1}, x_{2}, T\right) \\
\quad-\left\{\int_{0}^{T} \log \left|B(T)-B(t)-x_{3}\right| N_{3}\left(x_{1}, x_{2}, d t\right)\right. \\
\left.\quad-\int_{0}^{T} \int_{0}^{u} \frac{B(u)-B(t)-x_{3}}{\left|B(u)-B(t)-x_{3}\right|^{2}} N_{3}\left(x_{1}, x_{2}, d t\right) \cdot d B(u)\right\} .
\end{gathered}
$$

Since

$$
\begin{aligned}
N_{3}\left(x_{1}, x_{2}, d t\right)= & \frac{1}{\pi} \log \frac{1}{\left|x_{1}\right|} \tilde{\alpha}_{2}\left(x_{2}, d t\right) \\
& +\frac{1}{\pi} \log \frac{1}{\left|x_{2}\right|} \tilde{\alpha}_{2}\left(x_{1}, d t\right) \\
& +\frac{1}{\pi} \log \frac{1}{\left|x_{1}\right|} \log \frac{1}{\left|x_{2}\right|} d t,
\end{aligned}
$$

and

$$
\tilde{\alpha}_{2}=\alpha_{2}-N_{2}, \quad N_{2}(x, d t)=\frac{1}{\pi} \log \frac{1}{|x|} d t,
$$

we see that the last term of (5.5) is equal to

$$
\begin{gathered}
\frac{1}{\pi} \log \frac{1}{\left|x_{1}\right|}\left\{\int_{0}^{T} \log \left|B(T)-B(t)-x_{3}\right| \alpha_{2}\left(x_{2}, d t\right)\right. \\
\left.\quad-\int_{0}^{T} \int_{0}^{u} \frac{B(u)-B(t)-x_{3}}{\left|B(u)-B(t)-x_{3}\right|^{2}} \alpha_{2}\left(x_{2}, d t\right) \cdot d B(u)\right\} \\
+\frac{1}{\pi} \log \frac{1}{\left|x_{2}\right|}\left\{\int_{0}^{T} \log \left|B(T)-B(t)-x_{3}\right| \alpha_{2}\left(x_{1}, d t\right)\right. \\
\left.\quad-\int_{0}^{T} \int_{0}^{u} \frac{B(u)-B(t)-x_{3}}{\left|B(u)-B(t)-x_{3}\right|^{2}} \alpha_{2}\left(x_{1}, d t\right) \cdot d B(u)\right\} \\
+\frac{1}{\pi^{2}} \log \frac{1}{\left|x_{1}\right|} \log \frac{1}{\left|x_{2}\right|}\left\{\int_{0}^{T} \log \left|B(T)-B(t)-x_{3}\right| d t\right. \\
\left.-\int_{0}^{T} \int_{0}^{u} \frac{B(u)-B(t)-x_{3}}{\left|B(u)-B(t)-x_{3}\right|^{2}} d t \cdot d B(u)\right\}
\end{gathered}
$$




$$
\begin{aligned}
& =\frac{1}{\pi} \log \frac{1}{\left|x_{1}\right|}\left\{\pi \alpha_{3}\left(x_{2}, x_{3}, T\right)-\log \frac{1}{\left|x_{3}\right|} \alpha_{2}\left(x_{2}, T\right)\right\} \\
& \quad+\frac{1}{\pi} \log \frac{1}{\left|x_{2}\right|}\left\{\pi \alpha_{3}\left(x_{1}, x_{3}, T\right)-\log \frac{1}{\left|x_{3}\right|} \alpha_{2}\left(x_{1}, T\right)\right\} \\
& \quad+\frac{1}{\pi^{2}} \log \frac{1}{\left|x_{1}\right|} \log \frac{1}{\left|x_{2}\right|}\left\{\pi \alpha_{2}\left(x_{3}, T\right)-\log \frac{1}{\left|x_{3}\right|} T\right\},
\end{aligned}
$$

by (5.4) with $\alpha_{3}$ and $\alpha_{4}$ being replaced by $\alpha_{2}$ and $\alpha_{3}$.

Substituting $\alpha_{3}\left(x_{2}, x_{3}, T\right)=\tilde{\alpha}_{3}\left(x_{2}, x_{3}, T\right)+N_{3}\left(x_{2}, x_{3}, T\right)$ etc. into the above display, we see that the right-handed side of (5.5) is equal to

$$
\begin{aligned}
\pi \alpha_{4}\left(x_{1}, x_{2}, x_{3}, T\right)-\log \frac{1}{\left|x_{1}\right|} \tilde{\alpha}_{3}\left(x_{2}, x_{3}, T\right)-\log \frac{1}{\left|x_{2}\right|} \tilde{\alpha}_{3}\left(x_{1}, x_{3}, T\right) \\
\quad-\log \frac{1}{\left|x_{3}\right|} \tilde{\alpha}_{3}\left(x_{1}, x_{2}, T\right)-\frac{1}{\pi} \log \frac{1}{\left|x_{1}\right|} \log \frac{1}{\left|x_{2}\right|} \tilde{\alpha}_{2}\left(x_{3}, T\right) \\
\quad-\frac{1}{\pi} \log \frac{1}{\left|x_{1}\right|} \log \frac{1}{\left|x_{3}\right|} \tilde{\alpha}_{2}\left(x_{2}, T\right)-\frac{1}{\pi} \log \frac{1}{\left|x_{2}\right|} \log \frac{1}{\left|x_{3}\right|} \tilde{\alpha}_{2}\left(x_{1}, T\right) \\
\quad-\frac{1}{\pi^{2}} \log \frac{1}{\left|x_{1}\right|} \log \frac{1}{\left|x_{2}\right|} \log \frac{1}{\left|x_{3}\right|} T
\end{aligned}
$$

which we shall denote by $\pi \tilde{\alpha}_{4}\left(x_{1}, x_{2}, x_{3}, T\right)$. This is just the form $\tilde{\alpha}_{4}$ which we intend to find.

Now, we consider the left-handed side of (5.5). The following two multiparameter functionals are well-defined for $T>0,0<\varepsilon \leqslant 1$ and $x_{i} \in R^{2}$

$$
\begin{aligned}
& \Phi_{1}\left(T, \varepsilon, x_{1}, x_{2}, x_{3}\right)=\int_{0}^{T} \log \left(\left|B(T)-B(t)-x_{3}\right|^{2}+\varepsilon^{2}\right)^{1 / 2} \tilde{\alpha}_{3}\left(x_{1}, x_{2}, d t\right) \\
& \Phi_{2}\left(T, \varepsilon, x_{1}, x_{2}, x_{3}\right)=\int_{0}^{T} \int_{0}^{u} \frac{B(u)-B(t)-x_{3}}{\left|B(u)-B(t)-x_{3}\right|^{2}+\varepsilon^{2}} \tilde{\alpha}_{3}\left(x_{1}, x_{2}, d t\right) \cdot d B(u) .
\end{aligned}
$$

According to Rosen [17], to show that the left-handed side of (5.5) is jointly continuous in $T$ and $x_{1}, x_{2}, x_{3}$ it suffices to show that for any even integer $m$

$$
\begin{aligned}
& E\left|\Phi_{i}\left(T, \varepsilon, x_{1}, x_{2}, x_{3}\right)-\Phi_{i}\left(T^{\prime}, \varepsilon^{\prime}, x_{1}^{\prime}, x_{2}^{\prime}, x_{3}^{\prime}\right)\right|^{m} \\
& \quad \leqslant C_{m, \alpha}\left|\left(T, \varepsilon, x_{1}, x_{2}, x_{3}\right)-\left(T^{\prime}, \varepsilon^{\prime}, x_{1}^{\prime}, x_{2}^{\prime}, x_{3}^{\prime}\right)\right|^{m \alpha},
\end{aligned}
$$

where $\alpha$ is some positive real number independent on $m$. However, since the above display involves the higher moments of random variables, it seems that we cannot establish it by merely using white noises analysis, 
although the latter does have helped us to prove (1.2).

Finally, we mention that the renormalizations of $\alpha_{r}(x, T)$ have also been investigated by Dynkin [3] and Rosen [19]. We do not know whether these seemingly different renormalizations are in fact mutually inducible.

Add in the proof. It has been found that the arguments for proving Proposition 3.1, especially Lemma 3.1, are not perfectly right. The corrections together with some improvements will appear in "A W.N.C. Viewpoint on Intersection Local Times" (Proceedings on Gaussian Random Fields Conference, World Scientific 1991).

\section{REFERENCES}

[1] Dvoretzky, A., Erdös, P. and Kakutani, S., Multiple points of paths of Brownian motion in the plane, Bull. Res. Council Israel, F3 (1954), 364-371.

[2] Dynkin, E. B., Self-intersection guage for random walks and for Brownian motion, Ann. Probab., 16 (1988), 1-57.

[ 3 ] — Regularized self-intersection local times of planar Brownian motion, Ann. Probab., 16 (1988), 58-74.

[4] Geman, D., Horowitz, J. and Rosen, J., A local time analysis of intersections of Brownian paths in the plane, Ann. Probab., 12 (1984), 86-107.

[ 5] Hida, T., Analysis of Brownian Functionals, Carleton Math. Lecture Notes, no. 13 (1975), Carleton Univ. Ottawa.

[6] —-, Generalized multiple Wiener integrals, Proc. Japan Acad., 54A (1978), 5558.

[7] — B Brownian Motion, Application of Mathematics, vol. 11 (1980), SpringerVerlag.

[8] - Generalized Brownian functions and stochastic integrals, Appl. Math. Optim., 12 (1984), 115-123.

[ 9 ] - Lévy's Functional Analysis and Stochastic Analysis, Nagoya Univ. Lecture Note, (1986).

[10] Kallianpur, G. and Kuo, H. -H., Regularity property of Donsker's delta function, Appl. Math. Optim., 12 (1984), 89-95.

[11] Kubo, I., Ito formula for generalized Brownian functionals, Lecture Notes in Control and Information Sciences, vol. 49 (1983), 156-166, Springer-Verlag.

[12] Kuo, H. -H., Donsker's delta function as a generalized Brownian functionals and its application, Lecture Notes in Control and Information Sciences, vol. 49 (1983), 167-178 Springer-Verlag.

[13] Kuo, H. -H. and Shieh, N. R., A generalized Ito's formula for multi-dimensional Brownian motions and its applications, Chinese J. Math., 15 (1987), 163-174.

[14] Major, P., Multiple Wiener-Ito Integrals, Lecture Notes in Math., vol. 849 (1981), Springer-Verlag.

[15] Rosen, J., Self intersections of random fields, Ann. Probab., 12 (1984), 108-119.

[16] - A representation for the intersection local time of Brownian motion in space, Ann. Probab., 13 (1985), 145-153.

[17] —- Tanaka's formula for multiple intersections of planar Brownian motion, Stoch. Processes and Appl., 23 (1986), 131-141. 
[18] — Tanaka's formula and renormalization for intersections of planar Brownian motion, Ann. Probab., 14 (1986), 1245-1251.

[19] —- A renormalized local time for multiple intersections of planar Brownian motion, Lecture Notes in Math., vol. 1204 (1986), 515-531.

[20] Rosen, J. and Yor, M., Tanaka formulae and renormalization for triple intersections of Brownian motion in the plane, preprint 1988.

[21] Szegö, G., Orthogonal Polynomians, Amer. Math. Soc. Colloq. Publ., vol. XXIII (1939).

[22] Varadhan, S. R. S., Appendix to Euclidean Quantum field theory by K. Symanzik. In: Local Quantum Theory (R. Jost, ed.) A.P. 1969.

[23] Yor, M., Comploments aux formules des Tanaka-Rosen, Lecture Notes in Math., vol. 1123 (1986), 332-349, Springer-Verlag.

Department of Mathematics

National Taiwan University

Taipei, Taiwan 